PROCEEDINGS OF THE

AMERICAN MATHEMATICAL SOCIETY

Volume 130, Number 4, Pages 1221-1223

S 0002-9939(01)06126-3

Article electronically published on August 29, 2001

\title{
EPIMORPHISM SEQUENCES BETWEEN HYPERBOLIC 3-MANIFOLD GROUPS
}

\author{
TERUHIKO SOMA
}

(Communicated by Ronald A. Fintushel)

\begin{abstract}
We will show that any infinite sequence of epimorphisms between finitely generated hyperbolic 3-manifold groups eventually consists of isomorphisms.
\end{abstract}

In this paper, we are interested in sequences $M_{0} \stackrel{f_{0}}{\longrightarrow} M_{1} \stackrel{f_{1}}{\longrightarrow} \cdots \stackrel{f_{n-1}}{\longrightarrow} M_{n} \stackrel{f_{n}}{\longrightarrow} \cdots$ of $\pi_{1}$-surjective maps between geometric 3 -manifolds and the problem whether such a sequence has a homotopy equivalence. Our theme here is closely connected with J. Simon's Problem 1.12 (C) and Y. Rong's Problem 3.100 (B) in [5], and related topics are studied in [4, 6], 7], 8], 9], 10], 11], etc. We will consider the case where these 3-manifolds $M_{i}$ are hyperbolic. If such an $M_{i}$ has finite volume, then either $M_{i}$ is a closed manifold or each end of $M_{i}$ is a $\mathbb{Z} \times \mathbb{Z}$-cusp, and the fundamental group $\pi_{1}\left(M_{i}\right)$ is finitely generated. Example $3.2(2)$ in Reid-Wang-Zhou [7] presented a closed hyperbolic 3-manifold $M$ which admits, for any $n \in \mathbb{N}$, a length $n$ sequence $M_{0} \stackrel{f_{0}}{\longrightarrow} M_{1} \stackrel{f_{1}}{\longrightarrow} \cdots \stackrel{f_{n-1}}{\longrightarrow} M_{n}$ of non-homotopy equivalence, $\pi_{1}$-surjective maps between closed hyperbolic 3 -manifolds $M_{i}(i=0,1, \ldots, n)$ with $M_{0}=M$. On the other hand, Theorem 1 below shows that any infinite sequence of $\pi_{1}$-surjective maps between such 3-manifolds contains a homotopy equivalence.

Theorem 1. Suppose that

$$
G_{0} \stackrel{\varphi_{0}}{\longrightarrow} G_{1} \stackrel{\varphi_{1}}{\longrightarrow} G_{2} \stackrel{\varphi_{2}}{\longrightarrow} \cdots \stackrel{\varphi_{n-1}}{\longrightarrow} G_{n} \stackrel{\varphi_{n}}{\longrightarrow} \cdots
$$

is an infinite sequence of epimorphisms between the fundamental groups $G_{n}(n=$ $0,1,2, \ldots)$ of orientable hyperbolic 3 -manifolds (possibly of infinite volume). If $G_{0}$ is finitely generated, then $\varphi_{n}$ is isomorphic for all but finitely many $n \in \mathbb{N}$.

To prove Theorem 1, we use the character variety $X(G)$ of representations from a finitely generated group $G$ to $\mathrm{SL}_{2}(\mathbb{C})$ defined by Culler-Shalen [2, $\S 1$. Fix a generator set $\gamma_{1}, \ldots, \gamma_{\nu}$ for $G$, and let $\sigma(G)=\left\{g_{1}, \ldots, g_{\mu}\right\}$ be a maximal set such that each $g_{j}$ has a form $\gamma_{i_{1}} \cdots \gamma_{i_{r}} \in G$ for distinct positive integers $i_{1}, \ldots, i_{r} \leq \nu$. By [2, Proposition 1.4.1], the element $\chi_{\rho}$ of $X(G)$ corresponding to $\rho: G \longrightarrow \mathrm{SL}_{2}(\mathbb{C})$ can be identified with the point $\left(\operatorname{tr}\left(\rho\left(g_{1}\right)\right), \ldots, \operatorname{tr}\left(\rho\left(g_{\mu}\right)\right)\right) \in \mathbb{C}^{\mu}$, where $\operatorname{tr}\left(\rho\left(g_{j}\right)\right)$ is the trace of the $2 \times 2$ matrix $\rho\left(g_{j}\right)$. Then, $X(G)$ is a closed affine algebraic set in $\mathbb{C}^{\mu}$. We refer to Hartshorne [3] for the fundamental notation and definitions on algebraic geometry.

Received by the editors July 11, 2000 and, in revised form, September 27, 2000.

1991 Mathematics Subject Classification. Primary 57M50, 57M05.

Key words and phrases. Hyperbolic 3-manifolds, character varieties. 
Proof of Theorem [1. Let $A$ be the set of infinite sequences $\mathbf{n}=\left(n_{0}, n_{1}, n_{2}, \ldots\right)$ such that all entries $n_{i}$ are non-negative integers and at most finitely many of them are non-zero. We equip $A$ with the backward lexicographical order. That is, $\mathbf{n}=\left(n_{0}, n_{1}, n_{2}, \ldots\right)<\mathbf{m}=\left(m_{0}, m_{1}, m_{2}, \ldots\right)$ means that $n_{j_{0}}<m_{j_{0}}$ for some $j_{0} \in \mathbb{N} \cup\{0\}$ and $n_{j}=m_{j}$ for all $j \geq j_{0}+1$. Then, $A$ is a well ordered set. For a finitely generated group $G$, let $\alpha(G)$ be the element of $A$ such that the $i$-th term of $\alpha(G)$ is the number of $i$-dimensional irreducible components of $X(G)$.

Let $\varphi: G \longrightarrow H$ be an epimorphism between finitely generated groups. Then, the induced map $\varphi^{*}: X(H) \longrightarrow X(G)$ is an injective regular map. Here, $\varphi^{*}$ being regular means that it is the restriction to $X(H)$ of a polynomial map between the affine complex spaces containing $X(H)$ and $X(G)$. In fact, by identifying $\operatorname{tr}\left(\rho\left(\varphi\left(g_{j}\right)\right)\right)$ with $\operatorname{tr}\left(\left(\varphi^{*} \rho\right)\left(g_{j}\right)\right)$ for $\rho \in X(H)$ and $g_{j} \in \sigma(G)$, one can consider that $X(H)$ is an algebraic subset of $X(G)$ in $\mathbb{C}^{\mu}$. If an irreducible component $C$ of $X(H)$ is contained in an irreducible component $D$ of $X(G)$, then $\operatorname{dim}(C) \leq \operatorname{dim}(D)$. Moreover, if $\operatorname{dim}(C)=\operatorname{dim}(D)$, then we have $C=D$. This implies that $\alpha(G) \geq$ $\alpha(H)$, and the equality $\alpha(G)=\alpha(H)$ holds if and only if $X(G)=X(H)$.

Here, we return to an infinite sequence

$$
G_{0} \stackrel{\varphi_{0}}{\longrightarrow} G_{1} \stackrel{\varphi_{1}}{\longrightarrow} \cdots \stackrel{\varphi_{n-1}}{\longrightarrow} G_{n} \stackrel{\varphi_{n}}{\longrightarrow} \cdots
$$

given in the statement of Theorem 1 . Since $A$ is well ordered, there exists $n_{0} \in \mathbb{N}$ with $\alpha\left(G_{n_{0}}\right)=\alpha\left(G_{n}\right)$ for all $n \geq n_{0}$. Since $X\left(G_{n}\right)=X\left(G_{n+1}\right)$ if $n \geq n_{0}$, there exists $\chi_{\rho} \in X\left(G_{n+1}\right)$ with $\varphi_{n}^{*} \chi_{\rho}=\chi_{\widetilde{\eta}_{n}}$, where $\widetilde{\eta}_{n}: G_{n} \longrightarrow \mathrm{SL}_{2}(\mathbb{C})$ is a lift of a holonomy $\eta_{n}: G_{n} \longrightarrow \operatorname{Isom}^{+}\left(\mathbb{H}^{3}\right)=\mathrm{PSL}_{2}(\mathbb{C})$ of a hyperbolic 3-manifold $M_{n}$ with $\pi_{1}\left(M_{n}\right)=G_{n}$. Since $\widetilde{\eta}_{n}=\rho \circ \varphi_{n}$ is a faithful representation, $\varphi_{n}$ is monic and hence isomorphic. This completes the proof.

A knot $K$ in $S^{3}$ is called hyperbolic if the complement $S^{3}-K$ admits a hyperbolic structure of finite volume. Note that, for $G=\pi_{1}\left(S^{3}-K\right), X(G)$ has no 0 -dimensional irreducible component. Moreover, it is very often the case that the dimension of $X(G)$ is one. For example, if an exterior $E(K)$ for $K$ has no closed essential surface, then $\operatorname{dim} X(G)=1$ (see Cooper et al. 11, §2.4, Proposition]). Theorem 2 below is a knot-group version of Theorem 1 , where epimorphisms are not necessarily assumed to be peripheral preserving.

Theorem 2. Let $K_{0}$ be a hyperbolic knot in $S^{3}$ with $\operatorname{dim} X\left(G_{0}\right)=1$ and $n\left(K_{0}\right) \in \mathbb{N}$ the number of irreducible components of $X\left(G_{0}\right)$ for $G_{0}=\pi_{1}\left(S^{3}-K_{0}\right)$. Suppose that

$$
G_{0} \stackrel{\varphi_{0}}{\longrightarrow} G_{1} \stackrel{\varphi_{1}}{\longrightarrow} G_{2} \stackrel{\varphi_{2}}{\longrightarrow} \cdots \stackrel{\varphi_{n-1}}{\longrightarrow} G_{n}
$$

is a finite sequence of epimorphisms between the fundamental groups $G_{i}$ of the complements of hyperbolic knots $K_{i}$ in $S^{3}(i=1,2, \ldots, n)$ starting from $G_{0}$. If the length $n$ of the sequence is not less than $n\left(K_{0}\right)$, then at least one of these epimorphisms $\varphi_{j}$ is an isomorphism.

Proof. Let $a_{1}\left(G_{i}\right)$ be the number of (1-dimensional) irreducible components of $X\left(G_{i}\right)$. Suppose that any $\varphi_{i}(i=0,1, \ldots, n-1)$ are not isomorphic. Then, by the argument similar to that in Theorem 1, we have $a_{1}\left(G_{i}\right)>a_{1}\left(G_{i+1}\right)$. Since $a_{1}\left(G_{0}\right)=n\left(K_{0}\right)$ and $a_{1}\left(G_{n}\right) \geq 1$, the inequality $n<n\left(K_{0}\right)$ holds. 


\section{REFERENCES}

1. D. Cooper, M. Culler, H. Gillet, D.D. Long and P.B. Shalen, Plane curves associated to character varieties of 3-manifolds, Invent. Math. 118 (1994), 47-84. MR 95g:57029

2. M. Culler and P.B. Shalen, Varieties of group representations and splitting of 3-manifolds, Ann. of Math. 117 (1983), 109-146. MR 84k:57005

3. R. Hartshorne, Algebraic Geometry, Graduate Texts in Mathematics vol 52, Springer-Verlag, New York Heiderberg Berlin, 1977. MR 57:3116

4. C. Hayat-Legrand, S. Wang and H. Zieschang, Any 3-manifold 1-dominates at most finitely many Seifert manifolds with finite fundamental groups, Peking University Research Report No. 82, (1999), preprint.

5. R. Kirby, Problems in low-dimensional topology, Geometric Topology (W.H. Kazez ed.), AMS/IP Studies in Advanced Mathematics vol. 2, Part 2, Amer. Math. Soc. and International Press, 1997, pp. 35-473. CMP 98:01

6. A.W. Reid and S. Wang, Non-Haken 3-manifolds are not large with respect to mappings of non-zero degree, Comm. Anal. Geom. 7 (1999), 105-132. MR 2000c:57042

7. A.W. Reid, S. Wang and Q. Zhou, Generalized Hopfian property, minimal Haken manifold, and J. Simon's conjecture for 3-manifold groups, E-print: math.GT/0002003.

8. Y. Rong, Degree one maps between geometric 3-manifolds, Trans. Amer. Math. Soc. 332 (1992), 411-436. MR 92j:57007

9. T. Soma, Non-zero degree maps to hyperbolic 3-manifolds, J. Differential Geom. 49 (1998), 517-546. MR 2000b:57034

10. T. Soma, Sequences of degree-one maps between geometric 3-manifolds, Math. Ann. 316 (2000), 733-742. MR 2001b:57039

11. S. Wang and Q. Zhou, Any 3-manifold 1-dominates at most finitely many geometric 3manifolds, E-print: math.GT/0001058.

Department of Mathematical Sciences, School of Science and Engineering, Tokyo

Denki University, Hatoyama-Machi, Saitama-Ken 350-0394, Japan

E-mail address: soma@r.dendai.ac.jp 